\title{
Simulation Et Implémentation D'un Système PV sous l'Environnement Proteus Avec Nouvelle Commande MPPT
}

\author{
CHELLAKHI Abdelkhalek, ELBEID Said. Abouelmahjoub Younes.
}

\begin{abstract}
Résumé-Ce papier a pour objectif de valoriser une nouvelle technique MPPM (MPPT) pour extraire le maximum de puissance générer par le panneau photovoltaïque. L'environnement Proteus est utilisé pour implémenter et simuler la nouvelle approche MPPM grâce à la carte Arduino UNO et un afficheur LCD. Les résultats de simulation prouvent la meilleure performance de notre commande MPPM par rapport à les deux traditionnelles techniques P\&O et INC de dépister le point de puissance maximale PPM sous des conditions de changement brusque de l'éclairement solaire.
\end{abstract}

Mots-clés - Arduino UNO, Générateur PV, Nouvelle MPPM (MPPT), PPM, Proteus.

\section{INTRODUCTION}

L ENERGIE Photovoltaïque connaît aujourd'hui un fort développement dans le monde et au Maroc. Ce développement est dopé par les politiques internationales et nationales qui visent la diminution de l'utilisation des énergies polluantes et non renouvelables (fossiles). [1]

Pour cette raison, plusieurs pays prennent la démarche de s'orienter vers l'exploitation des énergies renouvelables, ils ont donné naissance à de grands projets qui sont basés sur les énergies durables et non épuisables. Et la grande partie de ces projets est consacré pour l'énergie photovoltaïque.

Malgré à son faible coût et sa simplicité dans l'installation, l'énergie photovoltaïque reste moins rentable, à cause de la fluctuation de sa production, qui est le résultat de la nonlinéarité de l'intensité lumineuse et la température. Par conséquent, la puissance maximale qui peut générer le panneau PV ne coïncide pas toujours avec son point de fonctionnement. Et pour remédier à ce problème, les chercheurs sont proposés des mécanismes (commandes, algorithmes) de poursuite de la puissance maximale (MPPM) ou en anglais MPPT (Maximum Power Point Tracker), qui a pour but d'extraire le maximum de puissance qui peut délivrer le générateur photovoltaïque dans les différentes conditions climatiques. Ses commandes nécessitent un convertisseur DC-DC entre le générateur PV et la charge pour adapter et contrôler la puissance délivrée par le

CHELLAKHI Abdelkhalek \& ABOUELMAHJOUB Younes are with National School of Applied Sciences of El Jadida. Chouaib-Doukkali University. (e-mail: chellakhi.fsjucd@yahoo.fr). panneau PV et la puissance qui nécessite la charge [2-3]. Et puis le MPP s'atteint par le contrôle de la durée d'impulsion du convertisseur D (Rapport cyclique) par l'algorithme MPPM.

Parmi les commandes MPPM les plus rencontrés dans la littérature, on trouve perturbe et observe (P\&O) [3-4-5], Incrémentation de la Conductance (INC) [4-5] et Hill-Climbing (HC) [6-7], ces trois méthodes sont catégorisées dans les commandes dites traditionnelles. Et il y a autres commandes comme la Logique Floue (LF) [3-4-8], Réseau des neurones (RN) [4-7] dite intelligentes.

Notre travail est une tentative de simplifier la modélisation et l'implémentation d'un système PV à l'aider d'un simple mais l'un des puissants logiciels qui donne la possibilité de l'implémentation complète d'un système PV. Et cela va simplifier la tâche pour mieux comprendre la procédure de travail d'un tel système $\mathrm{PV}$.

Notre travail est enchainé comme suit, le deuxième paragraphe est consacré pour étudier les différents composants d'un système PV ; le premier sous-paragraphe a été réservé pour la présentation du générateur $\mathrm{PV}$, alors que le deuxième sousparagraphe a été réservé pour étudier le convertisseur élévateur continu-continu (DC-DC). Et le dernier sous-paragraphe a été consacré pour présenter le principe de la nouvelle commande ainsi que les deux commandes $\mathrm{P} \& \mathrm{O}$ et INC. Dans le troisième paragraphe nous présentons les résultats de simulation pour évaluer la performance de la nouvelle commande MPPM ; le premier sous-paragraphe a été réservé pour la modélisation du générateur PV sous l'environnement Proteus, alors que dans le deuxième sous-paragraphe nous avons implémenté notre nouvelle commande MPPM, ainsi que les deux traditionnelles commandes P\&O et INC sur le contrôleur ATMega328 de l'Arduino UNO avec l'afficheur LCD. Au final nous avons terminé par une conclusion.

ELBEID Said is with National School of Applied Sciences of Marrakech. Cadi Ayyad University. (e-mail: elbeidsaid@yahoo.fr). 


\section{Modelisation D'Un Systeme PhotovoltaïQue}

\section{A. Générateur PV}

Le générateur PV est constitué de plusieurs cellules PV qui sont connectées en série ou/et en parallèle afin de produire le courant et la tension choisis. La Fig. 1 présente l'un des circuits équivalents à une cellule PV. L'expression mathématique qui décrit le courant produit par cette cellule est donnée par l'équation suivante [9] :

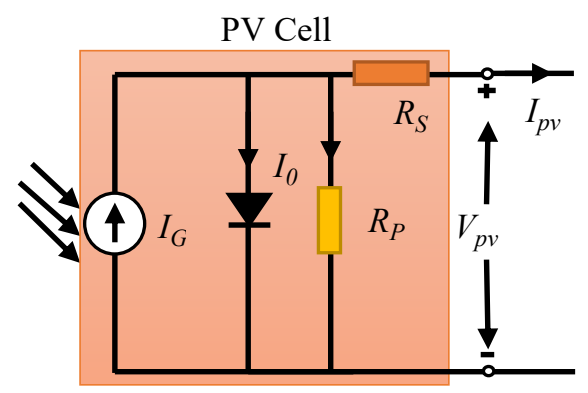

Fig. 1. Circuit équivalent d'une cellule PV.

$$
\begin{aligned}
I_{p V}= & N_{P} \times I_{G}-N_{P} \times I_{D}\left(\exp \left(\frac{q \times\left(V_{p V}+I_{p v} \times R_{S}\right)}{A \times K \times T \times N_{S}}\right)-1\right)- \\
& N_{P} \times \frac{q\left(V_{p V}+I_{p V} \times R_{S}\right)}{N_{S} \times R_{P}}
\end{aligned}
$$

Où, $\mathrm{I}_{\mathrm{pv}}$ est le courant de sortie du cellule $\mathrm{PV}, \mathrm{I}_{\mathrm{G}}$ est le photocourant, $\mathrm{I}_{0}$ représente le courant inverse de saturation de la diode, $\mathrm{N}_{\mathrm{P}}$ et $\mathrm{N}_{\mathrm{S}}$ sont le nombre des cellules en parallèle et en série, respectivement. q est la charge électronique $(1.6 \times$ $\left.10^{-19} \mathrm{C}\right), \mathrm{V}_{\mathrm{pv}}$ est la tension au borne du cellule PV, A est le facteur d'idéalité, $k$ et la constant de Boltzmann $(8.65 \times$ $\left.10^{-5} \mathrm{eV} / K\right)$ et $T$ est la température du jonction p-n $(K)$ [9].

Le photo courant du cellule PV est influencé par l'irradiation et la température ambiante, l'équation du $\mathrm{I}_{\mathrm{G}}$ est donnée comme suit :

$$
I_{G}=\left[I_{s} \times k_{i}\left(T+T_{r}\right)\right] \frac{G}{100}
$$

Où,

$\mathrm{k}_{\mathrm{i}}$ Est le coefficient de température.

$\mathrm{T}_{\mathrm{r}}$ Est la température de référence de la cellule $\mathrm{PV}\left({ }^{\circ} \mathrm{C}\right)$.

G Est l'irradiation solaire $\left(\mathrm{W} / \mathrm{m}^{2}\right)$.

$I_{S}$ Est le courant de saturation qui peut écrit sous la forme:

$$
I_{S}=I_{o S} \times\left(\frac{T}{T_{r}}\right)^{3} \times \exp \left(\left(-\frac{q \times E_{g}}{A \times K}\right) \times\left(\frac{1}{T_{T}}+\frac{1}{T}\right)\right]
$$

Où, $\mathrm{I}_{\mathrm{os}}$ est le courant inverse de saturation à $\mathrm{T}_{\mathrm{r}}$, et $\mathrm{E}_{\mathrm{g}}$ est l'énergie de gap du semi-conducteur de la cellule PV.

\section{B. Convertisseur Statique DC-DC}

Le convertisseur statique continu/continu (DC-DC) le plus fréquemment utilisé dans la conversion photovoltaïque est le convertisseur élévateur (booste), grâce à sa simplicité de leur circuit et de leur implémentation (Fig. 2) [10].

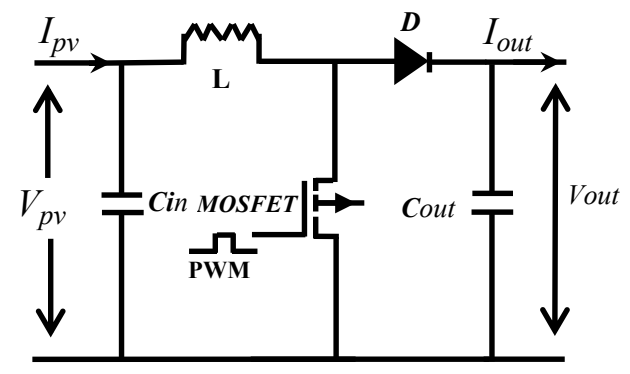

Fig. 2. Le convertisseur DC-DC élévateur (Boost).

La relation entre la tension à l'entrée du boost $V_{p v}$ et la tension de la sortie $V_{\text {out }}$ est donnée comme suit :

$$
\begin{gathered}
V_{o u t}=V_{p v} \frac{1}{1-D} \\
I_{o u t}=I_{p v}(1-D)
\end{gathered}
$$

Divisant (2) par (3), donne:

$$
R_{\text {out }}=\frac{V_{\text {out }}}{I_{\text {out }}}=\frac{1}{(1-D)^{2}} \times \frac{V_{p v}}{I_{p v}}=\frac{1}{(1-D)^{2}} \times R_{\text {in }}
$$

Où, $R_{\text {in }}$ et $R_{\text {out }}$ sont la résistance en entrée et en sortie du convertisseur respectivement. Dans un système PV, (4) pourra être écrite sur cette façon :

$$
R_{c h}=\frac{R_{p v}}{(1-D)^{2}}
$$

Où, $R_{c h}$ et la résistance de la charge, et $R_{p v}$ et la résistance voyait par le panneau PV.

D'après (5), la durée d'impulsion $\mathrm{D}$ (Le rapport cyclique) du convertisseur boost pourra être écrite comme suivant :

$$
D=1-\sqrt{\frac{R_{p v}}{R_{c h}}}
$$

\section{Mécanismes de Poursuite de Puissance Maximal (MPPM)}

1) Commandes $P \& O$ et INC.

Les deux commandes perturbe et observe $(\mathrm{P} \& \mathrm{O})$ et la incrémentation de la conductance sont les deux MPPM les plus utilisés dans le marché et aussi les plus rencontrés dans la littérature [3-5-7].

Le principe de la première commande $\mathrm{P} \& \mathrm{O}$ est très simple, et se résume dans la génération des perturbations au niveau de la tension du panneau PV en augmentant et en diminuant le rapport cyclique D du convertisseur DC-DC et d'observer l'effet de ses perturbations au niveau de la puissance produite par le générateur PV.

Pour la deuxième commande INC, son principe est basé sur la comparaison de l'opposé de la conductance $-\mathrm{G}=-\mathrm{I} / \mathrm{V}$ est l'incrément de la conductance $\mathrm{dG}=\mathrm{dI} / \mathrm{dV}$ pour en déduire la position du point de fonctionnement par rapport PPM. Si (dG) est supérieur à (-G), on diminue le rapport cyclique D. Par contre, si l'incrément de conductance est inférieur à l'opposé de la conductance, on augmente le rapport cyclique D. Ce processus est répété jusqu'à atteindre le PPM. 


\section{2) Nouvelle Commande MPPM}

Le principe de cette nouvelle commande MPPM est basé sur la zone des points de puissance maximale PPM qui est illustrée sur la Fig. 3 et la procédure de travail de cette commande est donnée par l'organigramme dans la Fig. 4.

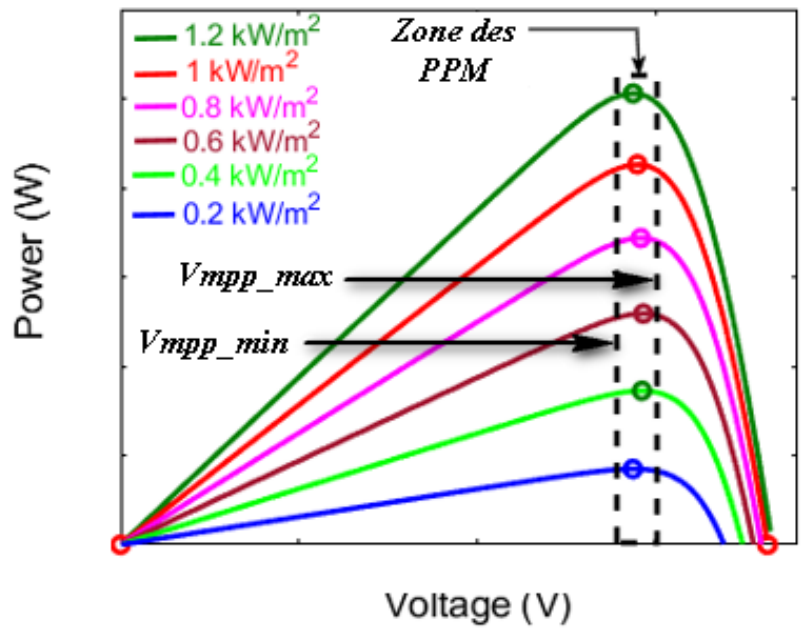

Fig. 3. Illustration du Zone du PPM dans la caractérisation P-V sous différentes irradiation.

On constate facilement d'après la Fig. 3 que la zone des puissances maximale est localisée par une région très étroite des tensions maximales $V_{m p p s}$ et avec deux limite $V_{m p p_{\text {min }}}$ et $V_{m p p_{\max }}$.

Premièrement, d'après la mesure du courant et la tension du générateur PV la tension $V(k)$ est comparé avec $V_{m p p_{\min }}$, et

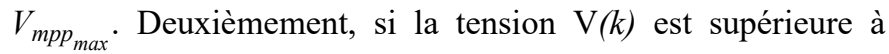
$V_{\text {mpp }_{\max }}$ elle reçoit la valeur du $V_{\text {mpp }}$ max , et si elle est inférieure à $V_{m p p_{\text {min }}}$, elle reçoit la valeur du $V_{m p p_{\text {min }}}$. Cette opération permet à la commande de focaliser la zone des PPM avec une grande précision et aussi avec une grande vitesse de convergence. Finalement, la relation (9) est utilisée afin de générer le nouveau rapport cyclique $D(k+1)$ :

$$
D(k+1)=1-\sqrt{\frac{V(k) / I(k)}{R_{\text {Load }}}}
$$

Mais, si la tension $V(k)$ est tombée dans la région $\left(V_{m p p_{\text {min }}}, V_{\text {mpp }}\right.$ max $)$, le nouveau rapport cyclique $D(k+1)$ va reçoit tout simplement le rapport cyclique de la derniere opération $D(k)$.

\section{Simulation Et Resultats}

La validation par simulation de notre nouvelle commande MPPM était faite sur l'environnement du logiciel Proteus, qui est l'un des logiciels de simulation le plus fréquemment utilisé, grâce à simplicité de travail et grâce les grandes bibliothèques des divers composants électroniques qui fournit. La carte Arduino UNO avec le microcontrôleur ATMega328 et parmi les composants qu'on peut utiliser avec souplesse sur l'environnement Proteus [11].

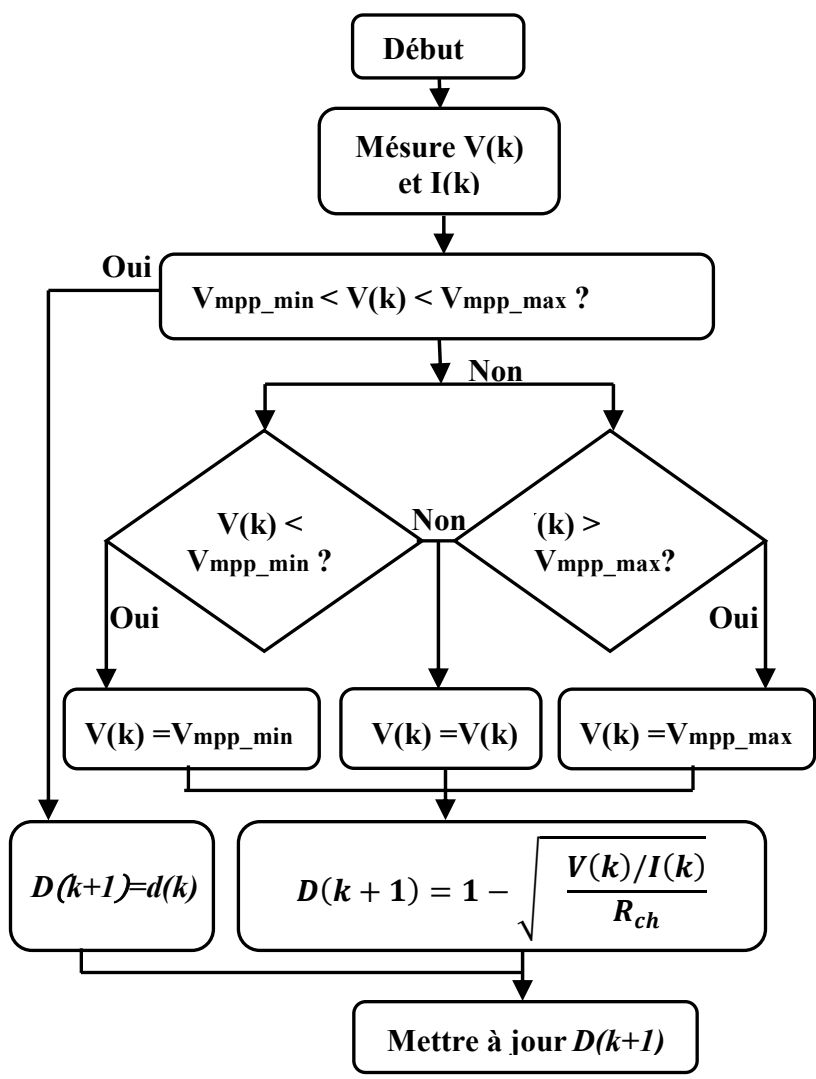

Fig. 4. Organigramme du MPPM proposée.

\section{A. Modulation du Générateur PV sur Proteus}

Le générateur PV que nous avons utilisé est simulé sur l'environnement Proteus et ses caractéristiques sont données dans le tableau I, et le circuit de générateur sur Proteus est illustré sur la Fig. 5 et le GPV en bloc avec le convertisseur DCDC est présenté par la Fig. 6 [11].

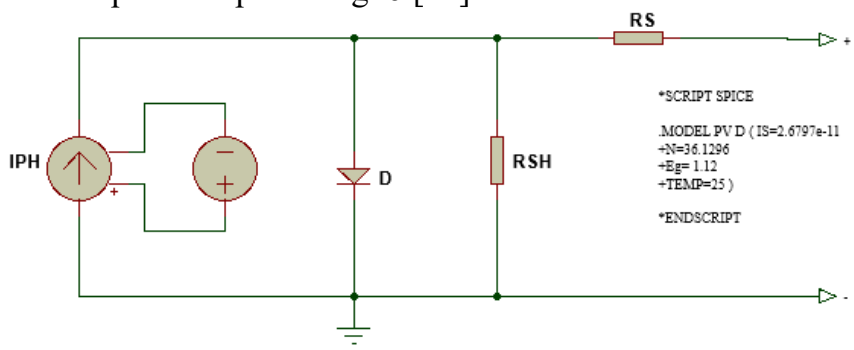

Fig. 5. Model d'un panneau PV sur Proteus.

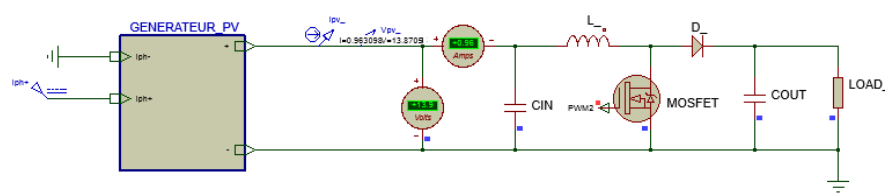

Fig. 6. Panneau PV avec le convertisseur DC-DC sous Proteus.

Les courbes de puissance en fonction de la tension P-V et de courant en fonction de la tension sous différente irradiation et à température constante $25^{\circ} \mathrm{C}$ sont présentés sur les deux figures Fig. 7 et Fig. 8. 
B. Implémentation de la nouvelle commande PPM sous Proteus avec ATMega 328 du Arduino UNO et avec afficheur $L C D$.

Nous avons implémenté notre nouvelle commande MPPM sur le contrôleur ATMega328 de l'Arduino UNO et pour mieux observer le changement de la puissance générer par le GPV, nous avons ajouté un afficheur LCD 16 bit.

Pour valoriser la robustesse de notre commande MPPM, nous avons la comparer avec les deux traditionnelles MPPM $\mathrm{P} \& \mathrm{O}$ et INC dans un premier temps sous les conditions STC et au final sous un changement brutal de l'irradiation solaire.

TABLE I. CARACTERISTIQUES DU GENERATEUR PV UTILISE A STC.
\begin{tabular}{|c|c|}
\hline Caractéristiques & Valeurs \\
\hline Point de Puissance Maximale PPM & $17 \mathrm{~W}$ \\
\hline Tension à PPM (Vppm) & $18.8 \mathrm{~V}$ \\
\hline Courant à PPM (Ippm) & $0.9 \mathrm{~A}$ \\
\hline Courant de court-circuit (Icc) & $1.01 \mathrm{~A}$ \\
\hline Tension de circuit ouvert (Vco) & 22.5 \\
\hline Coefficient de température de Vco (Kv) & $-0.35 \% /{ }^{\circ} \mathrm{C}$ \\
\hline Coefficient de température Icc (Ki) & $0.043 \% /{ }^{\circ} \mathrm{C}$ \\
\hline Photo-courant (Iph) & $1.173 \mathrm{~A}$ \\
\hline Courant de saturation (Is) & $2.6797 e-11 \mathrm{~A}$ \\
\hline Facteur d'idéalité & 1.0036 \\
\hline Résistance en parallèle (Rsh) & $405.96 \Omega$ \\
\hline Résistance en série (Rs) & $1.057 \Omega$ \\
\hline
\end{tabular}

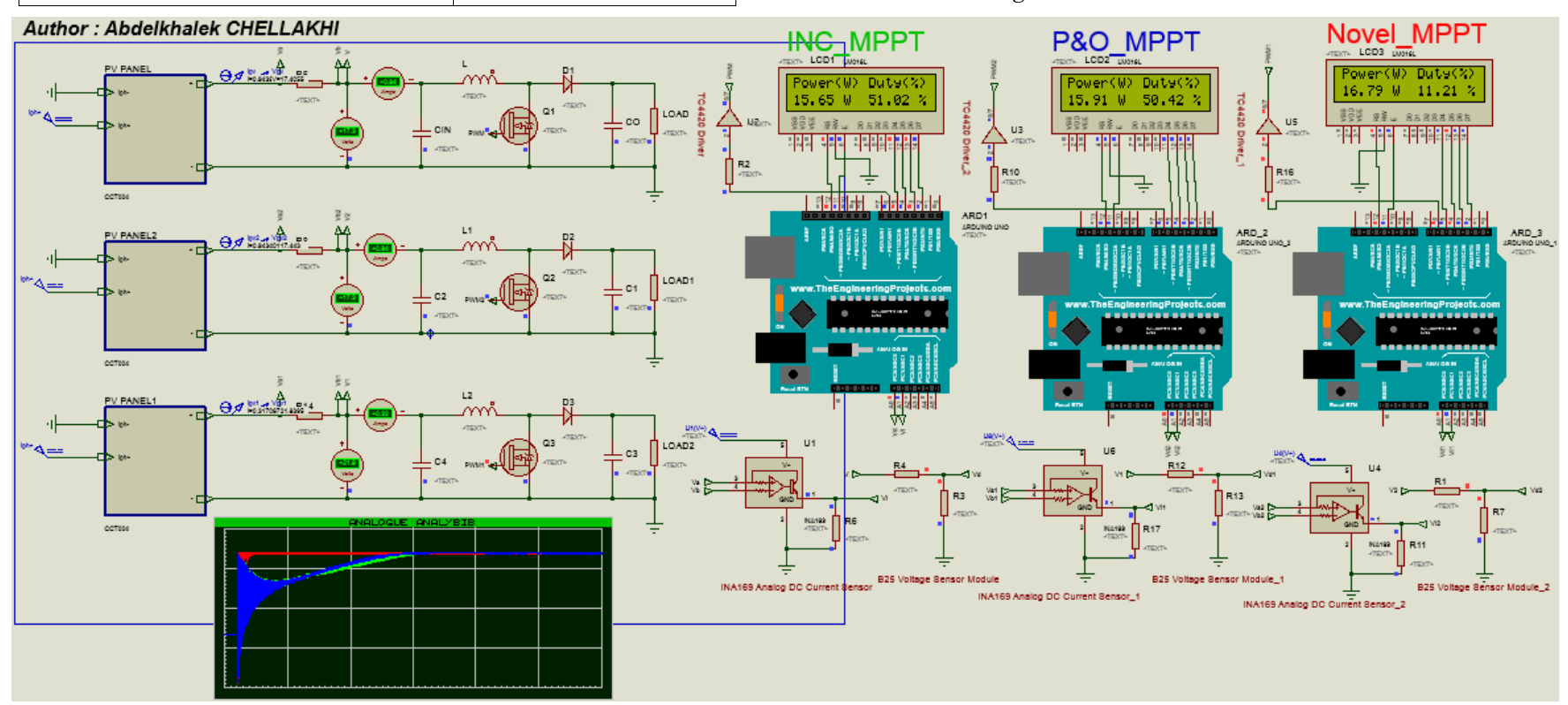

Fig. 9. Résultat de la simulation de la nouvelle commande MPPM comparait avec les deux traditionnelles MPPM P\&O et INC dans les conditions de test

standard (STC) $\left(1000 \mathrm{~W} / \mathrm{m}^{2}, T=25^{\circ} \mathrm{C}\right)$ sous l'environnement Proteus avec carte Arduino UNO et LCD.

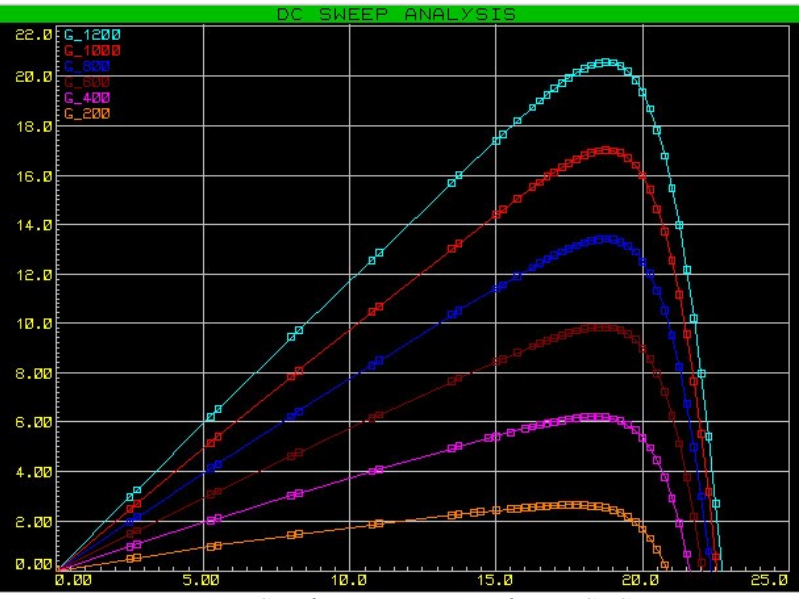

Fig. 7. Courbes P-V sous conditions STC.

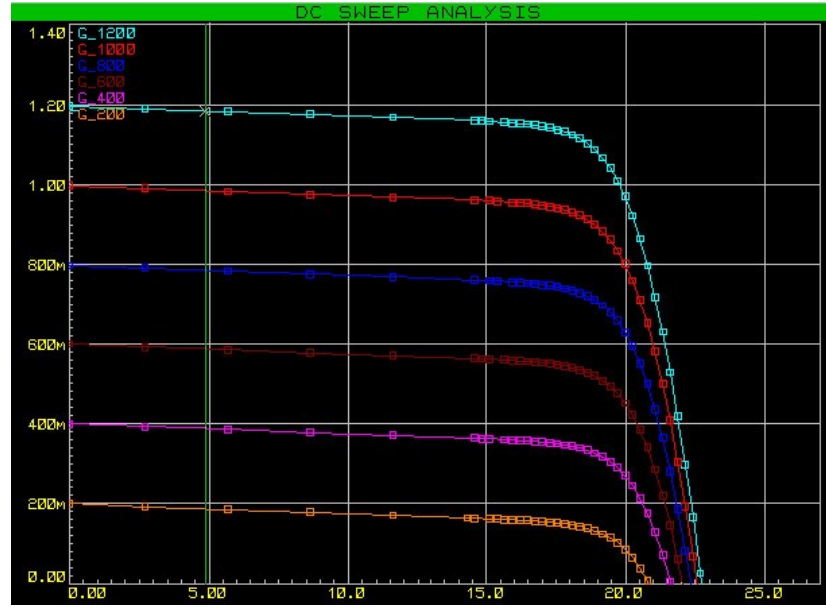

Fig. 8.Courbes $I-V$ sous conditions STC. 

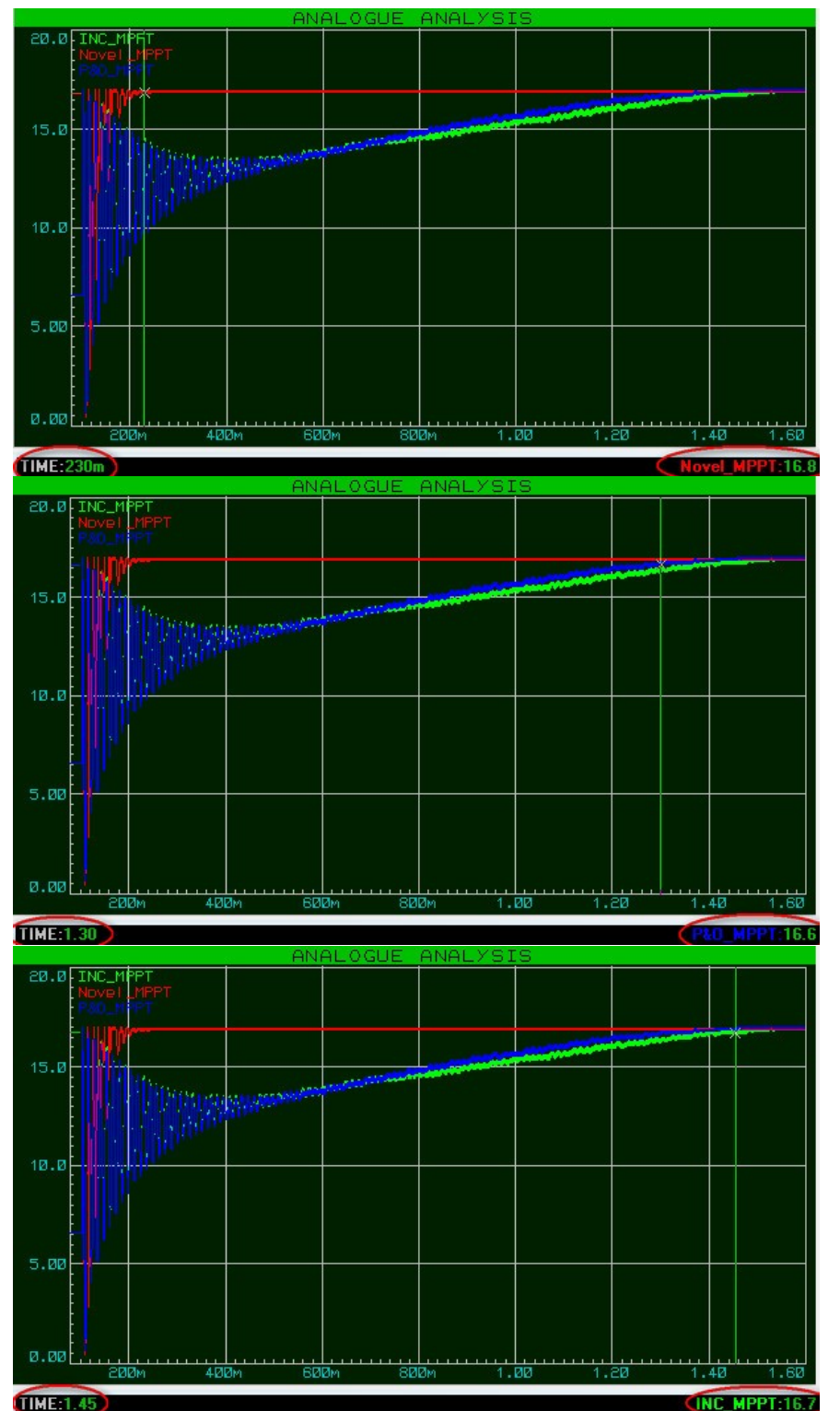

Fig. 10. Temps de convergence des trois commandes MPPM.

\section{1) Simulation et résultat sous les conditions STC.}

Dans cette simulation, nous avons implémenté trois GPV avec trois convertisseurs élévateurs (boost), et pour mesurer le courant et la tension nous avons utilisé un capteur de courant INA169 et un capteur de tension B25.

La fig. 9 présente l'implémentation des trois commandes MPPM sur trois contrôleurs ATMega328 de l'Arduino UNO, et avec des afficheurs LDC 16 bits.

La performance de notre commande est constatée d'après la Fig. 10, ou il est évident que notre approche MPPM permet de capter le point de puissance maximale (PPM) dans un temps qui ne dépasse pas même 0.23 microsecondes $(\mu \mathrm{s})$ et avec faible oscillation. Au contraire, les deux autres commandes $\mathrm{P} \& \mathrm{O}$ et INC n'atteignent pas le PPM sauf dans un temps qui dépasse $1.3 \mathrm{~s}$ et $1.45 \mathrm{~s}$ respectivement.

En plus, notre nouvelle approche MPPM peut atteint une efficacité de $98.82 \%$ ou lieu d'une efficacité de $97.64 \%$ et $98.23 \%$ pour les deux traditionnels algorithmes $\mathrm{P} \& \mathrm{O}$ et INC respectivement.

\section{2) Simulation et résultat sous un changement d'irradiation} solaire.

La deuxième simulation était faite pour objectif de tester la nouvelle commande MPPM suite à des changements brusques d'éclairement solaire, et aussi pour voir leur performance de suivre le PPM sous ces conditions.

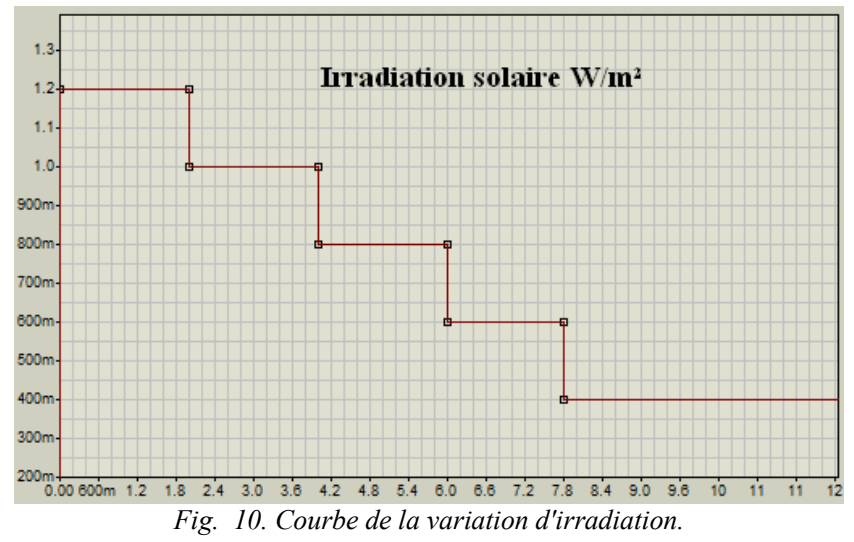

La fig. 11 présente la courbe de simulation de la variation soudaine d'irradiation solaire de $1200 \mathrm{~W} / \mathrm{m}^{2}$ à $400 \mathrm{~W} / \mathrm{m}^{2}$ avec une température constante de $25^{\circ} \mathrm{C}$.

Les résultats de simulation de la nouvelle commande comparés avec les deux autres algorithmes sont donnés par la fig. 12. Cette figure montre la meilleure performance de notre algorithme de suivre le PPM sous les brusques changements d'irradiation solaire, et aussi la grande vitesse de convergence vers le PPM. Par contre, les deux autres commandes sont influencées d'une manière très évidente lors des changements brusques de l'éclairement solaire. 


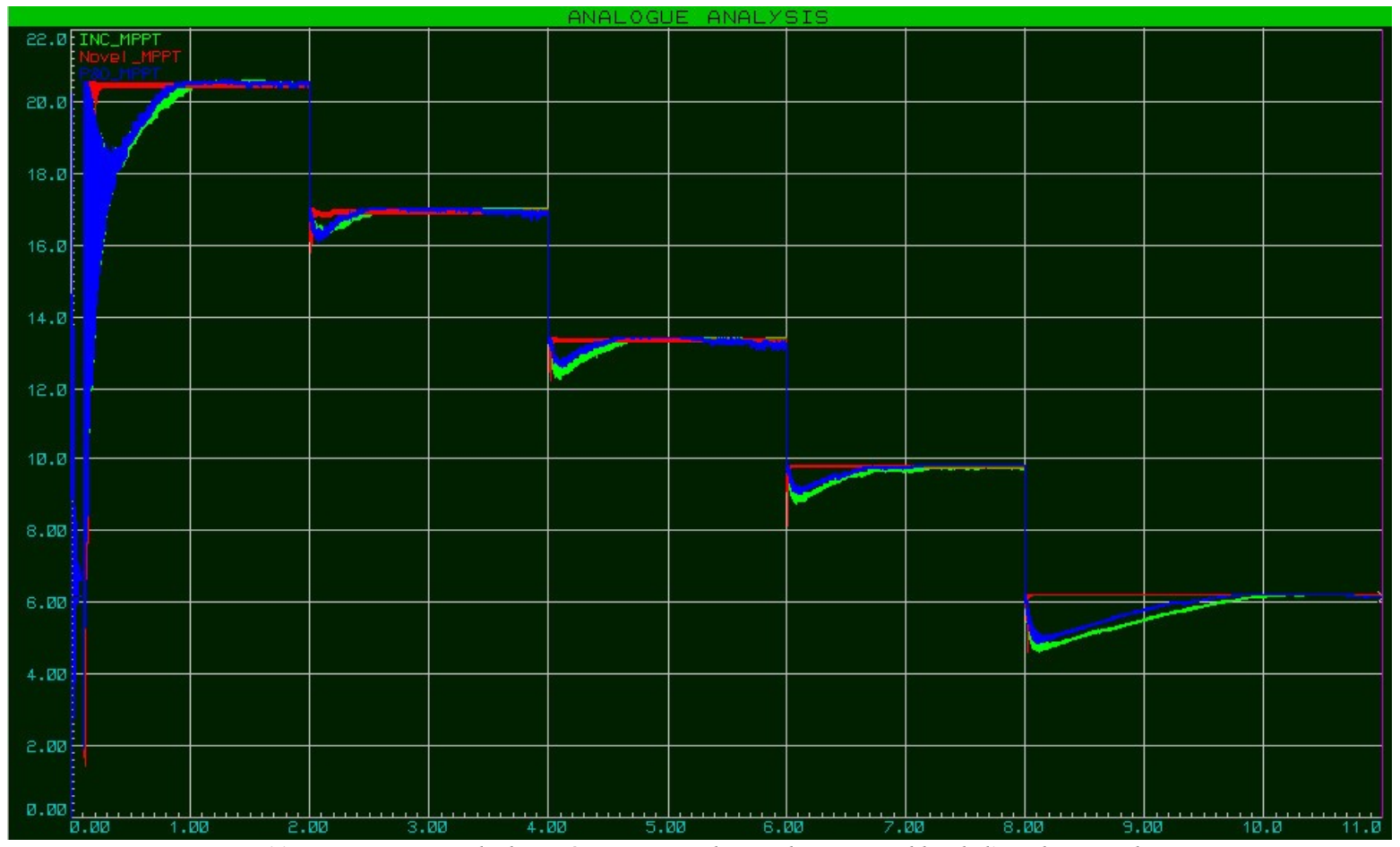

Fig. 11. Puissance optimale du système PV pour des conditions variables de l'irradiation solaire.

\section{CONCLUSION}

Ce travail était consacré d'abord pour une modélisation d'un générateur PV sous l'environnement Proteus. Ensuite, nous avons présenté brièvement le principe des deux commandes $\mathrm{P} \& \mathrm{O}$ et INC, et nous avons détaillé le principe de notre nouvelle commande MPPM.

Enfin, les résultats de simulation prouvent la validité et la grande performance de notre approche par rapport à les deux traditionnelles commandes dans les conditions de changement brusque de l'éclairement.

Nous avons adopté seulement la validation par simulation, on attendant une validation pratique comme perspective.

\section{REFERENCES}

[1] Stéphane Vighetti. Systèmes photovoltaïques raccordés au réseau : Choix et dimensionnement des étages de conversion. Sciences de l'ingénieur [physics]. Institut National Polytechnique de Grenoble INPG, 2010. Français.

[2] N. Prabaharan, K. Palanisamy, "Analysis and integration of multilevel inverter configuration with boost converters in a photovoltaic system" Energy Conversion and Management 128 (2016) 327-342

[3] Hanen Abbes, Hafedh Abid, Kais Loukil, Ahmad Toumi, Mohamed Abid, "Etude comparative de cinq algorithmes de commande MPPT pour un système photovoltaïque" Conférence Internationale des Energies Renouvelables (CIER'13), Sousse, Tunisie - 2013

[4] Boualem Bendib, Hocine Belmili, Fateh Krim, "A survey of the most used MPPT methods: Conventional and advancedalgorithms applied for photovoltaic systems". Renewable and Sustainable Energy Reviews 45 (2015) 637-648

[5] Nabil Karami, Nazih Moubayed, Rachid Outbib, "General review and classification of different MPPT Techniques" Renewable and Sustainable Energy Reviews 68 (2017) 1-18.

[6] Hwa-Dong Liu, Chang-Hua Lin, Kai-Jun Pai, Yu-Liang Lin, "A novel photovoltaic system control strategies for improving hill climbing algorithm efficiencies in consideration of radian and load effect", Energy Conversion and Management 165 (2018) 815-826.
[7] Mohamed Lasheen, Mazen Abdel-Salamc, "Maximum power point tracking using Hill Climbing and ANFIS techniques for PV applications: A review and a novel hybrid approach", Energy Conversion and Management 171 (2018) 1002-1019.

[8] Shahriar Farajdadian, S.M. Hassan Hosseini, "Design of an optimal fuzzy controller to obtain maximum power in solar power generation system", Solar Energy 182 (2019) 161-178

[9] Ali Khatibi, Fatemeh Razi, Astaraei, Mohammad Hossein Ahmadi, "Generation and combination of the solar cells: A current model" review, Energy Sci Eng. 2019;7:305-322.

[10] N. Prabaharan, K. Palanisamy, Analysis and integration of multilevel inverter configuration with boost converters in a photovoltaic system, Energy Conversion and Management 128 (2016) 327-342.

[11] Saad Motahhir, Abdelilah Chalh, Abdelaziz El Ghzizal, Aziz Derouich, Development of a Low-cost PV System using an improved INC algorithm and a PV panel Proteus model, Journal of Cleaner Production (2018), doi: 10.1016/j.jclepro.2018.08.246. 